\title{
Single versus double screw fixation for the treatment of scaphoid waist fractures: Finite element analysis and preliminary clinical results in scaphoid nonunion
}

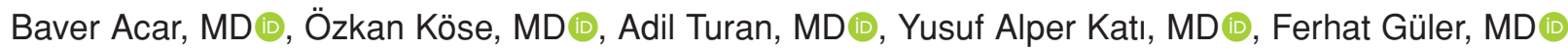 \\ Department of Orthopedics and Traumatology, University of Health Sciences, Antalya Training and Research Hospital, Antalya, Turkey
}

A scaphoid fracture is the most common carpal bone fracture that constitutes around $60 \%$ of all carpal fractures. ${ }^{[1]}$ Although stable, non-displaced and incomplete fractures can be treated conservatively, while displaced scaphoid fractures usually necessitate surgical fixation. ${ }^{[2]}$ Currently, centrally placed single headless compression screw fixation is accepted as a standard technique for the treatment of scaphoid fractures. ${ }^{[3]}$ Despite progression in the surgical techniques and development of better implants, nonunion in scaphoid fractures continues to be a challenging problem in hand surgery. In a previous meta-analysis, the nonunion rate in acute scaphoid fractures has been reported as high as $19 \%{ }^{[4]}$

Nonunion after scaphoid fractures is a multifactorial problem, and various factors may play a role in the development of nonunion. However, two crucial biological features of scaphoid bone among all other factors are vitally important. First is the vascular characteristic of the scaphoid bone. Secondly, $80 \%$ of the scaphoid surface is surrounded by cartilage; thus primary bone healing, also called Haversian bone healing, takes place without a callus

Received: September 26, 2019

Accepted: November 28, 2019

Published online: March 02, 2020

Correspondence: Baver Acar, MD. SBÜ Antalya Eğitim ve Arasstırma Hastanesi Ortopedi ve Travmatoloji Kliniği,

07100 Muratpaşa, Antalya, Türkiye.

E-mail: baveracar@hotmail.com

Doi: $10.5606 /$ ehc. 2020.71521

Citation: Acar B, Köse Ö, Turan A, Kałı YA, Güler F. Single versus double screw fixation for the treatment of scaphoid waist fractures: Finite element analysis and preliminary clinical results in scaphoid nonunion. Jt Dis Relat Surg 2020;31(1):73-80.

\section{ABSTRACT}

Objectives: This study aims to compare the single versus double screw fixation of scaphoid waist fractures using finite element analysis, and to present the preliminary clinical results of double screw fixation in a consecutive series of patients with scaphoid nonunion.

Patients and methods: A transverse scaphoid waist fracture (Herbert type B2) model was fixed with either single or double cannulated compression screws. Displacement and rotation of the fragments were performed using three-dimensional finite element analysis in three different wrist positions. A retrospective review was performed on 13 male patients (mean age 31.6 \pm 12.8 years; range, 17 to 64 years) who underwent double screw fixation for an established scaphoid nonunion in our clinic between January 2015 and December 2017. Assessment of union was established with serial plain radiographs in eight patients and with wrist computed tomography in five patients. Clinical evaluation was performed using the Mayo wrist score and visual analog scale (VAS).

Results: In all wrist positions, the displacement of the fracture gap in double screw fixation in all planes (x, y, and $\mathrm{z}$ ) was less than in single screw fixation. Similarly, rotation of the fracture fragments around the longitudinal axis of the scaphoid was lower in double screw fixation. Complete union was obtained in all patients. The mean time to union was $5 \pm 0.75$ months (range, 4 to 6 months). The mean VAS was $0.8 \pm 0.9$ (range, 0 to 3 ). Mayo wrist score was $91 \pm 6.9$ (range, 80 to 100 ) at the final follow-up.

Conclusion: Double-screw fixation technique may be a solution to reduce the rate of scaphoid nonunion in unstable type B2 scaphoid fractures or nonunion.

Keywords: Finite element analysis, scaphoid fracture, scaphoid nonunion, screw fixation.

formation. ${ }^{[5,6]}$ Furthermore, even under physiological loading in static wrist positions, the scaphoid bone is subjected to translational, bending, and rotational forces. As a result, in case of insufficient fixation, these forces may cause tearing of the newly-formed vasculature that leads to deterioration of the vascular supply and consequent nonunion. ${ }^{[5]}$ 
Some authors have claimed that single screw fixation does not provide sufficient rotational stability and proposed different fixation techniques to avoid this problem. Slade and Dodds ${ }^{[7]}$ recommended the placement of an additional K-wire along the single screw fixation to increase rotational stability. Biomechanical and clinical studies have shown that newly-developed scaphoid plates provide more stable fixation. ${ }^{[8,9]}$ In addition, Erhart et al. ${ }^{[10]}$ have developed new anti-rotational screws particularly designed for scaphoid fractures.

Recently, double screw fixation instead of the single screw has been advocated to increase the rotational stability. There are few biomechanical studies comparing the stability of single versus double screw fixation with conflicting results. ${ }^{[8,9]}$ Similarly, there are only two clinical studies that reported higher union rates in double screw fixation compared to single screw fixation in the current literature. ${ }^{[11,12]}$

We hypothesized that double screw fixation would provide greater rotational stability regarding biomechanical analysis and nonunion rates would be reduced. To the best of our knowledge, this topic has not been previously studied using finite element analysis. Therefore, in this study, we aimed to compare the single versus double screw fixation of scaphoid waist fractures using finite element analysis, and to present the preliminary clinical results of double screw fixation in a consecutive series of patients with scaphoid nonunion.

\section{PATIENTS AND METHODS}

This retrospective study was conducted at Antalya Training and Research Hospital between January 2015 and December 2017. In this study, threedimensional (3D) finite element stress analysis was utilized based on isotropic materials and static linear analysis in a computerized environment. On a complete transverse scaphoid fracture (Herbert classification, type B2) model, fixation was performed with a headless variable pitch compression screw in two different configurations from the volar approach (distal to proximal direction). In the first fixation technique, single $3.5 \mathrm{~mm}$ compression screw (Acutrak 2 Mini, Acumed, Hillsboro, OR, USA) fixation was applied, and in the second fixation technique, two parallel $2.5 \mathrm{~mm}$ compression screw (Acutrak 2 Micro, Acumed, Hillsboro, OR, USA) fixation was applied (Figure 1). The screw fixations were simulated parallel to the scaphoid long axis, and perpendicular to the fracture plane.
Normal physiological loading was applied in these models in loaded neutral $(\mathrm{N})$, maximum extension (ME), and maximum flexion (MF) static wrist positions. The normal physiological loads were calculated as described by Varga et al. ${ }^{[12]}$ These forces are described as a combination of the magnitude of the cartilage contact forces and ligament forces acting on the scaphoid. The contact pressures over the contact areas for each bone surrounding the scaphoid were integrated to compute the cartilage contact forces and the ligament forces on the scaphoid were computed according to the ligament insertion points.

Activity 880 optic scanner (smart optics Sensortechnik GmbH, Bochum, Germany), Rhinoceros 4.0 3D modeling software (3670 Woodland Park Ave N, Seattle, WA, USA), VRMesh Studio (Virtual Grid Inc., Bellevue City, WA, USA), and Algor Fempro analysis software (ALGOR Inc., 150 Beta Drive Pittsburgh, PA, USA) were used for the editing and optimization of the 3D surface mesh models, and solid meshing performing finite element analysis.

The models were created geometrically on VRMesh software and transferred to Algor Fempro software in stereolithography (STL) format (a universal file format for 3D modeling software) for analysis. The coordinates of the nodes were saved in the STL format so that part of an assembly could be transferred between different software.

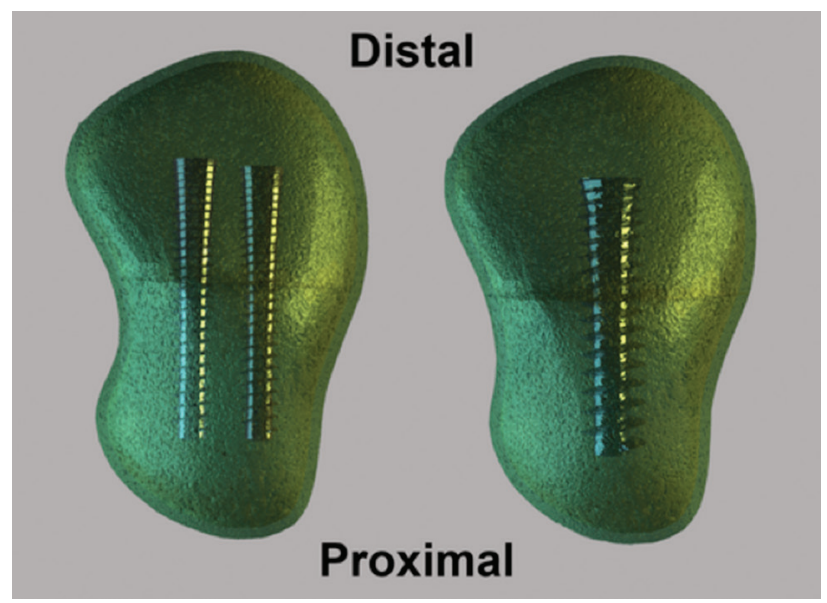

FIGURE 1. A complete transverse scaphoid fracture (Herbert classification, type B2) model was fixed in two different configurations: single or double headless variable pitch compression screw (Acutrack 2 Mini, Acutrack 2 Micro, Acumed, Hillsboro, OR, USA). 
The wrist modeling was based on data obtained from the "Visible Human Project" study. ${ }^{[13]}$ Axial scans adopted from that study were reconstructed in 3D-doctor software (Able Software Corporation, 5 Appletree Lane, Lexington, MA, USA) by extracting bone tissue. The 3D model was exported in STL format. The wrist modeling was applied using the mesh modeling technique. The models were converted to solid models as bricks and tetrahedral elements, and in this modeling system, Fempro uses eight-noded elements as far as possible. When the required detail could not be obtained with eight-noded elements, seven, six, five, and fournoded elements were used, respectively. The models used more than one million elements, and all the models were accepted as homogenous and isotropic materials. A homogenous material had the same mechanical properties in each structural element, and an isotropic material had uniformly the same material properties in all directions. Linear elasticity is the proportional variability of deformation of the structure.

The physical properties (Young's modulus and Poisson's ratio) of all the materials forming the model are shown in Table I. Friction contact interaction was defined between the bone surfaces in the fracture plane with the friction coefficient set to 0.4 at the fracture interface. ${ }^{[14]}$

The screws were 3D scanned by smart optics and saved in STL format. A transverse waist fracture was created, perpendicular to the long axis of the scaphoid (Herbert classification type B2). These files were then opened in Rhinoceros software, and the adaptation of the implants to the other sets was managed and transferred to Fempro analysis software with the $3 \mathrm{D}$ coordinates preserved. The fracture fixation was modeled in two different screw fixations, as single volar or double volar.

Loading was applied in three different static wrist positions of ME, MF, and N. No further loads were applied to the scaphoid in these wrist positions other than normal physiological loads. In each wrist

\begin{tabular}{|lcc|}
\hline \multicolumn{4}{c|}{ TABLE I } \\
\multicolumn{1}{|c|}{ Physical properties of all materials forming study model } \\
\hline Material & Young's modulus (Gpa) & Poisson's ratio \\
\hline Cortical bone & 13.7 & 0.3 \\
Cancellous bone & 1.0 & 0.3 \\
Titanium (screw) & 110.0 & 0.3 \\
\hline Gpa: Gigapascals. & & \\
\hline
\end{tabular}

position, the direction and magnitude of these loads change, as described in detail by Varga et al. ${ }^{[12]}$

In the control group, four different points were randomly identified on the fracture plane. In ME, $\mathrm{MF}$, and $\mathrm{N}$ positions, the maximum displacement was calculated separately at $\mathrm{x}, \mathrm{y}$, and $\mathrm{z}$ coordinates in these points. The point at which the maximum displacement occurred was selected, and this selected point was used as a guide in all other calculations in the fixation models (Figure 2).

Two symmetrical vectors were defined at the fracture interface in the proximal and distal fragments in the unloaded scaphoid models. In ME, MF and N, the loading rotational movements of these vectors were calculated to analyze the rotational displacements of the model (Figure 3).

A retrospective review was performed on 13 male patients (mean age $31.6 \pm 12.8$ years; range, 17 to 64 years) who underwent double screw fixation for an established scaphoid nonunion in Antalya Training and Research Hospital between January 2015 and December 2017. The mean follow-up
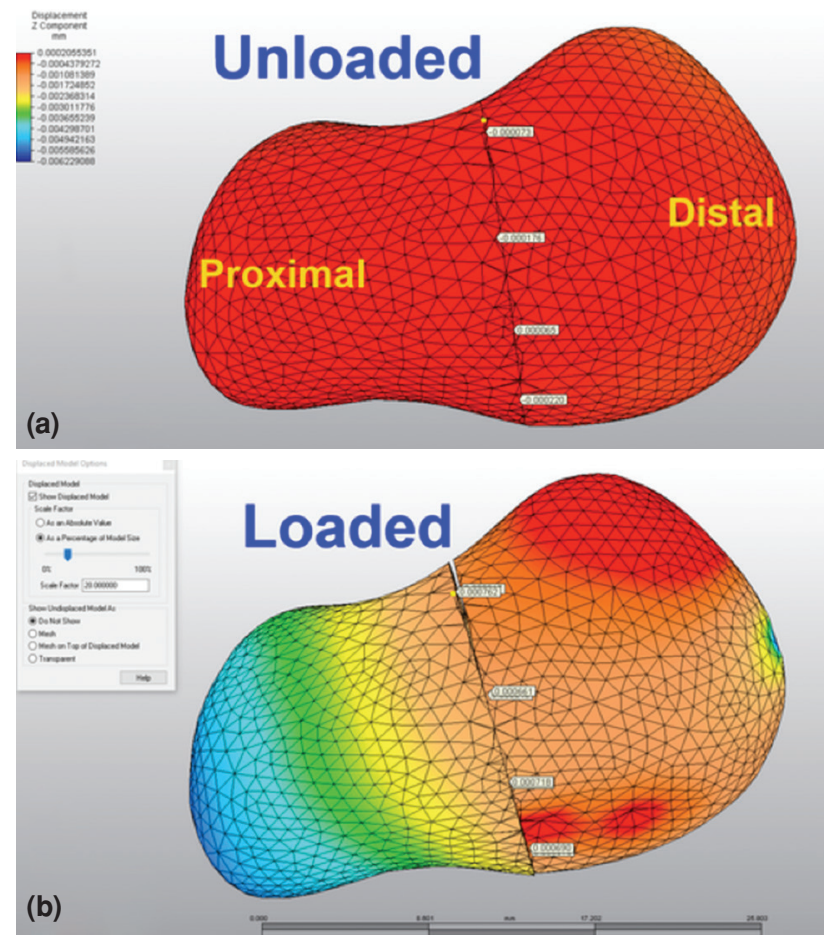

FIGURE 2. Schematic representation of calculation of displacement. (a) Four corresponding points were selected on fracture plane in unloaded model. (b) Displacement between these points was calculated in each wrist position and on each of $x, y$, and $z$ axes after loading. Maximally displaced point was set as guide for further tests. 

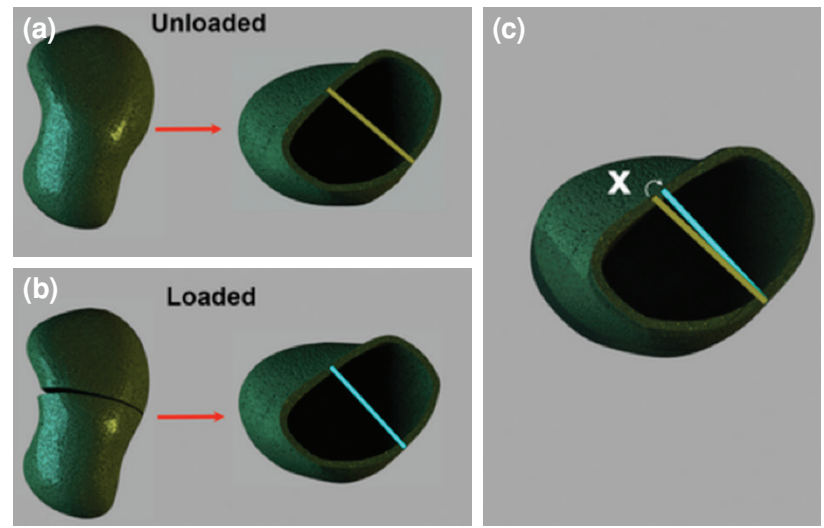

FIGURE 3. Schematic representation of calculation of rotation between proximal and distal fragments. (a) Two vectors were formed on plane of fracture in each fragment in unloaded model. (b) After loading, (c) relative rotation of vectors (yellow and blue lines) was calculated and recorded in degrees (represented by angle $\mathrm{x}$ ).

period was $23.2 \pm 9.2$ months (range, 7 to 39 months). The study protocol was approved by the Antalya Training and Research Hospital Ethics Committee. A written informed consent was obtained from each patient. The study was conducted in accordance with the principles of the Declaration of Helsinki.

A volar approach was used in all patients, and two headless cannulated compression screws (Acutrack 2 Micro, Acumed, Hillsboro, OR, USA) were used for the fixation of fractures. In five patients, tricortical iliac bone graft was used, and the remaining eight patients received autologous cancellous bone graft. Assessment of union was performed with serial plain radiographs. In case of suspicion, computed tomography (CT) was used to confirm the union. Union was accepted as the disappearance of the fracture line on three-view (posteroanterior, lateral, and scaphoid views) radiographs of the wrist and the observation of the trabecular bone bridging. Presence of cortical penetration of the screws was examined on the tomography images.

Functional outcome and pain were assessed with the Mayo wrist score and visual analog scale (VAS), respectively. Summary of clinical characteristics and functional results are summarized in Table II. All complications throughout the follow-up period were recorded such as revision surgery, infection, and wound problems. Furthermore, time to return to work, and the level of sporting activity reached after healing were evaluated.

\section{Statistical analysis}

No statistical analysis was possible as the values obtained from the finite element stress analysis emerged from mathematical calculations without any variance. The critical issues were the precise assessment and the amount and distribution of the stress on the scans.

\section{RESULTS}

In all wrist positions, the total displacement of the fracture gap in double screw fixation in all planes

\begin{tabular}{|c|c|c|c|c|c|c|}
\hline \multicolumn{7}{|c|}{$\begin{array}{l}\text { TABLE II } \\
\text { istics and fur }\end{array}$} \\
\hline Case\# & Age (year) & Gender & Grafting & $\begin{array}{l}\text { Follow-up } \\
\text { (months) }\end{array}$ & $\begin{array}{c}\text { VAS } \\
\text { (Final follow-up) }\end{array}$ & $\begin{array}{c}\text { Mayo wrist score } \\
\text { (Final follow-up) }\end{array}$ \\
\hline 1 & 22 & M & Tricortical & 39 & 0 & 100 \\
\hline 2 & 29 & M & Cancellous & 34 & 1 & 80 \\
\hline 3 & 29 & M & Cancellous & 31 & 2 & 90 \\
\hline 4 & 17 & M & Tricortical & 30 & 1 & 95 \\
\hline 5 & 41 & M & Cancellous & 29 & 0 & 90 \\
\hline 6 & 28 & M & Cancellous & 23 & 1 & 100 \\
\hline 7 & 46 & M & Cancellous & 23 & 1 & 90 \\
\hline 8 & 23 & M & Tricortical & 22 & 0 & 95 \\
\hline 9 & 64 & M & Cancellous & 19 & 0 & 80 \\
\hline 10 & 30 & $M$ & Cancellous & 17 & 0 & 95 \\
\hline 11 & 38 & M & Cancellous & 17 & 0 & 100 \\
\hline 12 & 22 & M & Tricortical & 11 & 1 & 90 \\
\hline 13 & 22 & M & Tricortical & 7 & 3 & 85 \\
\hline
\end{tabular}


$(\mathrm{x}, \mathrm{y}$, and $\mathrm{z})$ was lower than in single screw fixation (Table III). In each wrist position, the relative rotation of the fracture fragments around the longitudinal axis of the scaphoid bone was lower in double screw fixation (Table IV).
Complete bony union was obtained in all patients (Figure 4). The mean time to union was $5 \pm 0.8$ months (range, 4 to 6 months). The mean VAS was $0.8 \pm 0.9$ (range, 0 to 3 ) and the mean Mayo wrist score was $91 \pm 6.9$ (range, 80 to 100) at the final

\begin{tabular}{|c|c|c|c|}
\hline \multicolumn{4}{|c|}{$\begin{array}{c}\text { TABLE III } \\
\text { acement in } \mathrm{x}, \mathrm{y}, \mathrm{z} \text { ax }\end{array}$} \\
\hline Displacement $(\mu \mathrm{mm})$ & Maximum flexion & Maximum extension & Neutral \\
\hline \multicolumn{4}{|c|}{ Double volar screw fixation } \\
\hline$x$ & 0.029 & 0.186 & 0.030 \\
\hline y & 0.045 & 0.274 & 0.082 \\
\hline$z$ & 0.014 & 0.0830 & 0.056 \\
\hline \multicolumn{4}{|c|}{ Single volar screw fixation } \\
\hline$x$ & 0.068 & 0.26 & 0.031 \\
\hline$y$ & 0.26 & 0.282 & 0.086 \\
\hline z & 2.228 & 0.1250 & 0.095 \\
\hline
\end{tabular}

\section{TABLE IV}

Rotation between proximal and distal fragments

\begin{tabular}{|lccc|}
\hline Rotation angle $\left({ }^{\circ}\right)$ & Maximum flexion & Maximum extension & Neutral \\
\hline Double volar screw fixation & $\mathbf{0 . 0 0 1 2 7 4}$ & $\mathbf{0 . 0 0 3 3 5 8 6}$ & $\mathbf{0 . 0 0 0 1 0 6 8 5 2}$ \\
Single volar screw fixation & 0.001891 & 0.039531 & 0.000109686 \\
\hline Values are presented in degree. Bold numerals signify the lowest values. & & \\
\hline
\end{tabular}
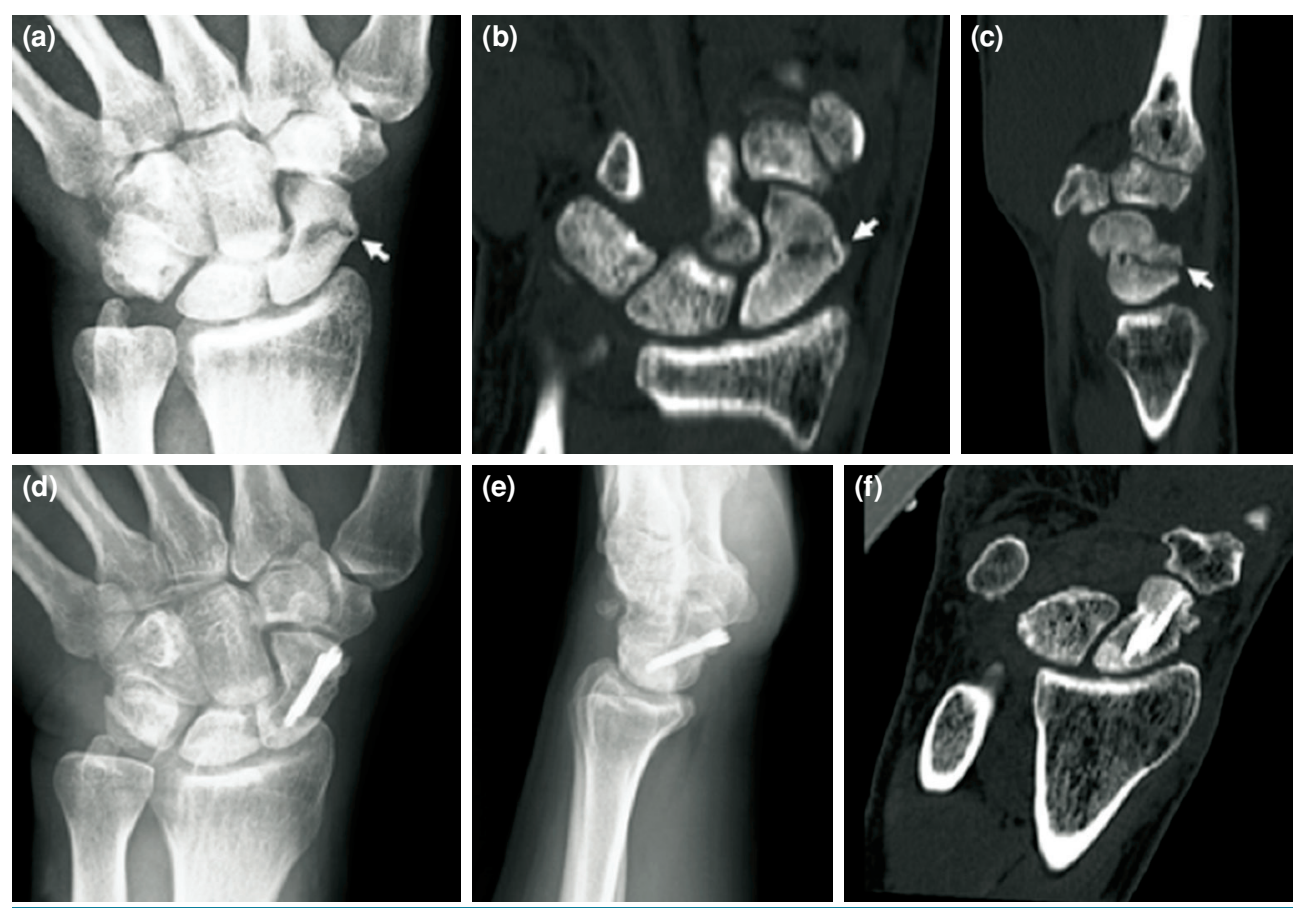

FIGURE 4. (Case 6\#) (a) Preoperative posteroanterior radiograph showing scaphoid nonunion (white arrow). Preoperative (b) coronal and (c) sagittal computed tomography (CT) scan of wrist (white arrows indicate nonunion). Postoperative (d) posteroanterior and (e) lateral radiographs at final follow-up 22 months after fixation. (f) Coronal CT view of scaphoid showed complete consolidation at final follow-up. 

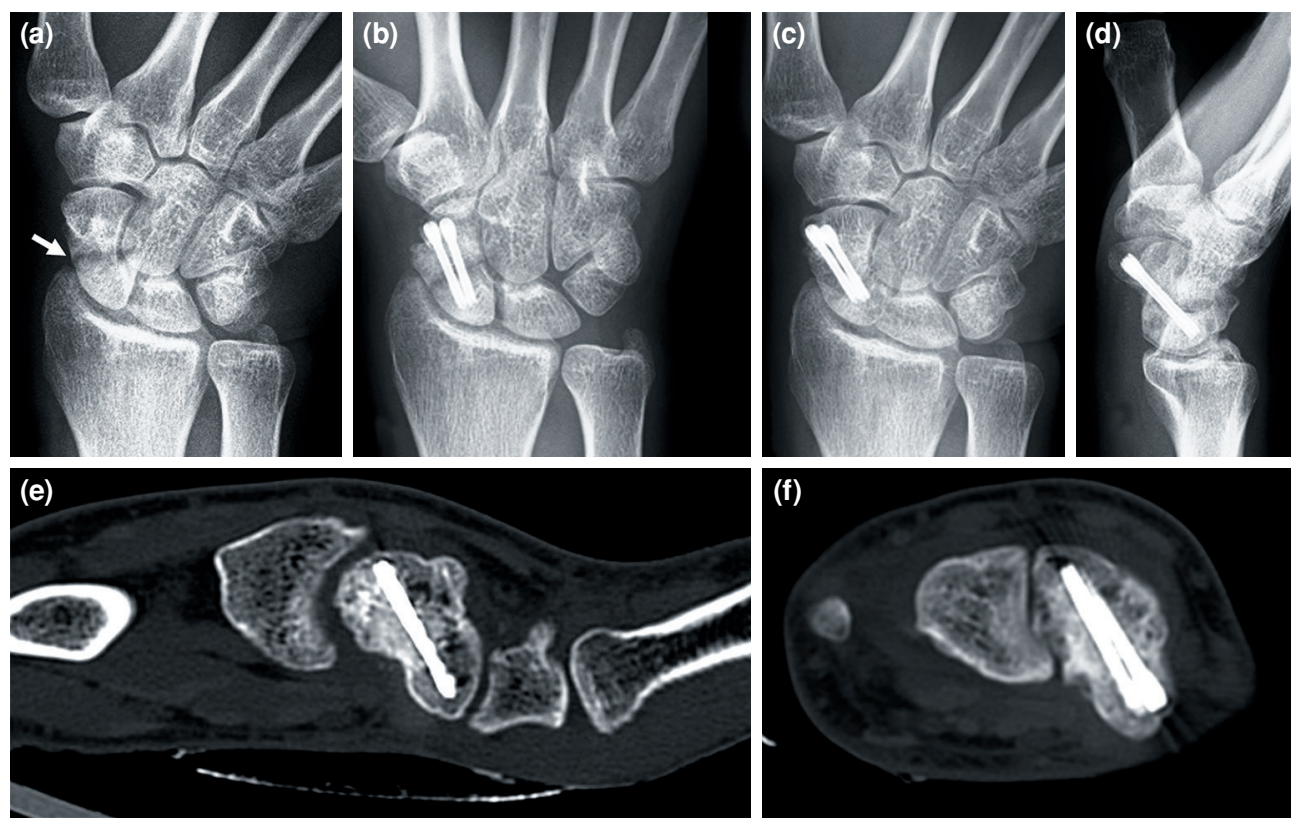

FIGURE 5. (Case 7\#) (a) Preoperative posteroanterior radiograph showing scaphoid nonunion (white arrow). (b) Early postoperative posteroanterior radiographs at second month follow-up. Postoperative (c) posteroanterior and (d) lateral radiographs at final follow-up 20 months after fixation. (e) Sagittal and (f) axial oblique computed tomography view of scaphoid showed complete consolidation at final follow-up.
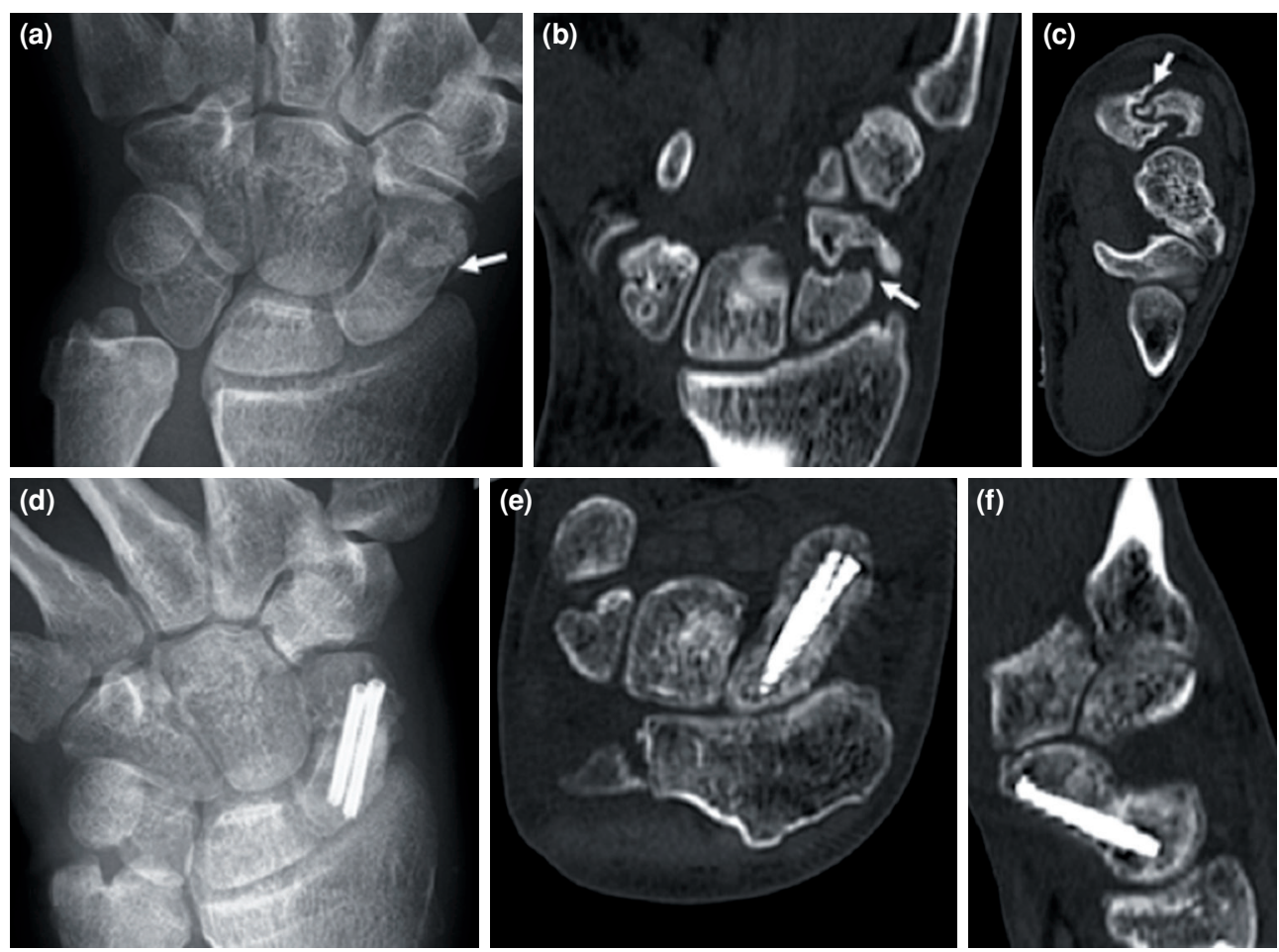

FIGURE 6. (Case 13\#) (a) Preoperative posteroanterior radiograph showing scaphoid nonunion (white arrow). Preoperative (b) coronal and (c) sagittal computed tomography (CT) scan of wrist (white arrows indicate nonunion). (d) Postoperative posteroanterior radiographs at final follow-up seven months after fixation. (e) Coronal and (f) sagittal CT view of scaphoid showed complete consolidation at final follow-up. 
follow-up (Table II). In five patients, where CT was taken, no cortical penetration of the screws was observed. No findings of screw loosening or migration were seen on the serial radiographs and final CT images (Figures 5 and 6).

No patients required revision surgery. No infection or complications were observed in any patient. All patients regained the preoperative level of working capability and sporting activity without any restriction.

\section{DISCUSSION}

In this study, single versus double screw fixation of scaphoid waist fractures was compared using a virtual computerized 3D finite element stress analysis method regarding rotation and displacement. In addition, the short-term clinical results of double screw fixation in a small consecutive case series were presented. The results of the finite element analysis showed that double screw fixation provides greater angular and rotational stability compared to single screw fixation in each tested static wrist position in all axes. In the clinical study, the union was achieved in all cases, and satisfactory functional outcomes were obtained without any significant complications. Based on the biomechanical and clinical results, double screw fixation can be suggested as a suitable option for scaphoid waist fractures and nonunion as it provides greater stability and high rate of union. ${ }^{[14]}$

Achieving union is a clinical problem in scaphoid fractures due to its anatomical and biomechanical characteristics. The scaphoid bone acts as a bridge between the wrist and the distal row carpal bones and is continuously exposed to shear forces. The scaphoid is nourished with a single vascular supply from the distal towards the proximal part. Thus, in case of a fracture, the vascularity of the proximal part is compromised. It is entirely intraarticular and covered with articular cartilage which limits the availability of the fixation techniques ${ }^{[5,6,15]}$ Advances in implant technology, such as the development of new screws and scaphoid plate designs providing compression, have significantly reduced the rates of scaphoid nonunion. Despite the range of these new techniques and implants used, the search for a more stable method for scaphoid bone fixation continues. ${ }^{[8]}$

Previously, few biomechanical studies have compared bone fixation with single versus double screws in scaphoid fractures, and the results of these studies provided conflicting conclusions.
Jurkowitsch et al. ${ }^{[9]}$ compared $2.2 \mathrm{~mm}$ double screw fixation, $2.2 \mathrm{~mm}$ single screw fixation, and $1.5 \mathrm{~mm}$ screw plate combinations and showed no difference between the plate and two screw combinations while both were superior to the single screw fixation. In contrast, Beutel et al. ${ }^{[8]}$ compared the four different combinations of scaphoid fixations; double screw fixation using two $1.5 \mathrm{~mm}$ screws, $2.2 \mathrm{~mm}$, and $3 \mathrm{~mm}$ single screw fixation, and $1.5 \mathrm{~mm}$ screw plate combination. They reported that the weakest fixation method was obtained with double screws of $1.5 \mathrm{~mm}$ diameter. This result is in contradiction with the results obtained both in our work and by Jurkowitsch. However, Buetel tried to explain this contradictory result indicating that the screws used in the double screw combination were thin $(1.5 \mathrm{~mm})^{[9,10]}$ Similar to the results of the study by Jurkowitsch et al., ${ }^{[9]}$ the results of the current study showed that double screw fixation was superior to single screw in respect of both displacement and rotation. Although the diameter of the single screw is thicker than the double screw's, it has been shown to be weaker than two thin screws, particularly against rotational forces.

The stability of implants used in scaphoid bone fractures is usually tested under laboratory conditions with forces applied at a single angle of the wrist until implant failure develops in cadavers. ${ }^{[16]}$ However, this testing methodology may not reflect the real physiological biomechanics and may not be valid for real conditions. For this reason, in this study, biomechanical comparisons were performed with the finite element analysis for mimicking physiological loadings.

To the best of our knowledge, there are two previous clinical studies in the English literature that have reported the outcomes of double screw fixation in scaphoid fractures. In a study of 19 patients (12 waist-type, five proximal third, and two distal third scaphoid fractures) by Garcia et al., ${ }^{[1]}$ fixation with double screws was reported to have resulted in $100 \%$ union. In another study by Quadlbaue et al. ${ }^{[5]}$ of 32 patients (22 single screw and 10 double screw fixations), a union rate of $86 \%$ (3 nonunions) was determined with single screw and $100 \%$ with double screw fixation. ${ }^{[12]}$ Absence of nonunion with double screw fixation in those two studies suggests the importance of absolute stability in scaphoid fractures. Similarly, in the current study, $100 \%$ union was achieved with double screw fixation. The reason behind these findings can be explained with increased stability. In fact, a single screw fixation is not without rotational stability since compression between the 
fracture planes creates secondary rotational stability due to the interdigitation of the rough bone surfaces and friction. However, it is well-known that rotational stability obtained at the time of initial fixation is lost over time due to the osteolysis at the fracture interface with a single screw. On the other hand, fixation with two screws provides persistent stability, maintains its stiffness even if the osteolysis develops.

This study has both strengths and limitations. Although the fracture was standardized, only one type of fracture model was tested, and in reality, there may be a variety of fracture types and/or comminution. Furthermore, the test results may be affected by morphological variations in the size and shape of the scaphoid. Screw fixation (parallel to the long axis of the scaphoid and perpendicular to the fracture plane) was also standardized; however, in reality, it may be challenging to achieve this optimal position. Moreover, physiological loads were simulated in only three static wrist positions, while ulnar and radial deviation, supination, and pronation were not taken into consideration. Nevertheless, subjective bias was eliminated with the use of computerized calculations in all models. In the clinical part of the study, the inclusion of a limited number of patients and the retrospective collection of data are the two significant limitations.

In conclusion, this is the first study that utilizes finite element modeling to compare single versus double screw fixation in scaphoid fractures. Our findings concluded that the double screw fixation construct was more stable than single screw fixation. In our limited retrospective case series, union was obtained in all patients. Double screw fixation technique may be a solution to reduce the rate of scaphoid nonunion by its contribution to rotational and angular stability in unstable type B2 scaphoid fractures. In the future, well-designed randomized clinical trials may further illuminate the problem and provide stronger evidence-based knowledge on this issue.

\section{Declaration of conflicting interests}

The authors declared no conflicts of interest with respect to the authorship and/or publication of this article.

\section{Funding}

This work was supported by the Turkish Ministry of Health (No: 81266704/774.99).

\section{REFERENCES}

1. Hove LM. Epidemiology of scaphoid fractures in Bergen, Norway. Scand J Plast Reconstr Surg Hand Surg 1999;33:423-6.

2. Lee SK. Fractures of the carpal bones. In: Wolfe SW, editor. Green's Operative Hand Surgery. 7th ed. Philadelphia: Elsevier; 2017. p. 594-5.

3. Dias J, Brealey S, Choudhary S, Cook L, Costa M, Fairhurst C, et al. Scaphoid Waist Internal Fixation for Fractures Trial (SWIFFT) protocol: a pragmatic multi-centre randomised controlled trial of cast treatment versus surgical fixation for the treatment of bi-cortical, minimally displaced fractures of the scaphoid waist in adults. BMC Musculoskelet Disord 2016;17:248.

4. Ferguson DO, Shanbhag V, Hedley H, Reichert I, Lipscombe S, Davis TR. Scaphoid fracture non-union: a systematic review of surgical treatment using bone graft. J Hand Surg Eur Vol 2016;41:492-500.

5. Quadlbauer S, Beer T, Pezzei C, Jurkowitsch J, Tichy A, Hausner T, et al. Stabilization of scaphoid type B2 fractures with one or two headless compression screws. Arch Orthop Trauma Surg 2017;137:1587-95.

6. Kawamura K, Chung KC. Treatment of scaphoid fractures and nonunions. J Hand Surg Am 2008;33:988-97.

7. Slade JF 3rd, Dodds SD. Minimally invasive management of scaphoid nonunions. Clin Orthop Relat Res 2006;445:108-19.

8. Beutel BG, Melamed E, Hinds RM, Gottschalk MB, Capo JT. Mechanical evaluation of four internal fixation constructs for scaphoid fractures. Hand (N Y) 2016;11:72-7.

9. Jurkowitsch J, Dall'Ara E, Quadlbauer S, Pezzei C, Jung I, Pahr D, et al. Rotational stability in screw-fixed scaphoid fractures compared to plate-fixed scaphoid fractures. Arch Orthop Trauma Surg 2016;136:1623-8.

10. Erhart J, Unger E, Schefzig P, Varga P, Trulson I, Gormasz A, et al. Rotational stability of scaphoid osteosyntheses: An in vitro comparison of small fragment cannulated screws to novel bone screw sets. PLoS One 2016;11:e0156080.

11. Garcia RM, Leversedge FJ, Aldridge JM, Richard MJ, Ruch DS. Scaphoid nonunions treated with 2 headless compression screws and bone grafting. J Hand Surg Am 2014;39:1301-7.

12. Varga P, Schefzig P, Unger E, Mayr W, Zysset PK, Erhart J. Finite element based estimation of contact areas and pressures of the human scaphoid in various functional positions of the hand. J Biomech 2013;46:984-90.

13. Available at: http:www.nlm.nih.gov/research/visible/ visiblehuman.html

14. Atik OŞ. Is there something new and interesting in my article? Eklem Hastalik Cerrahisi 2019;30:69.

15. Varga P, Zysset PK, Schefzig P, Unger E, Mayr W, Erhart J. A finite element analysis of two novel screw designs for scaphoid waist fractures. Med Eng Phys 2016;38:131-9.

16. Mandaleson A, Tham SK, Lewis C, Ackland DC, Ek ET. Scaphoid fracture fixation in a nonunion model: A biomechanical study comparing 3 types of fixation. J Hand Surg Am 2018;43:221-8. 